\title{
Detecting and Localizing Keyboard Accessibility Failures in Web Applications
}

\author{
Paul T. Chiou \\ University of Southern California \\ USA \\ paulchio@usc.edu
}

\author{
Ali S. Alotaibi \\ University of Southern California \\ USA \\ aalotaib@usc.edu
}

\author{
William G. J. Halfond \\ University of Southern California \\ USA \\ halfond@usc.edu
}

\begin{abstract}
The keyboard is the most universally supported input method operable by people with disabilities. Yet, many popular websites lack keyboard accessible mechanism, which could cause failures that make the website unusable. In this paper, we present a novel approach for automatically detecting and localizing keyboard accessibility failures in web applications. Our extensive evaluation of our technique on real world web pages showed that our technique was able to detect keyboard failures in web applications with high precision and recall and was able to accurately identify the underlying elements in the web pages that led to the observed problems.
\end{abstract}

\section{CCS CONCEPTS}

- Software and its engineering;

\section{KEYWORDS}

Web Accessibility, WCAG, Software Testing, Keyboard Navigation

\section{ACM Reference Format:}

Paul T. Chiou, Ali S. Alotaibi, and William G. J. Halfond. 2021. Detecting and Localizing Keyboard Accessibility Failures in Web Applications. In Proceedings of the 29th ACM foint European Software Engineering Conference and Symposium on the Foundations of Software Engineering (ESEC/FSE '21), August 23-28, 2021, Athens, Greece. ACM, New York, NY, USA, 13 pages. https://doi.org/10.1145/3468264.3468581

\section{INTRODUCTION}

The Web is fundamentally designed to work for "all people" [5]; yet, $15 \%$ of the world's population possesses some type of disability [28] that can hinder their ability to use the Web. For them, access to web applications is particularly important as the Web provides connections to services and information that might otherwise be unavailable [9]. Despite legislation that mandates companies to provide equally accessible websites [54], web accessibility issues are widespread. As of $2019,70 \%$ of internet sites contained "accessibility blockers" that made critical functionality inaccessible to disabled users [3], resulting in considerable disadvantages for people with disabilities.

Users with disabilities are often unable to use a traditional pointand-click or touch-operated device and must use other mechanisms

Permission to make digital or hard copies of part or all of this work for personal or classroom use is granted without fee provided that copies are not made or distributed for profit or commercial advantage and that copies bear this notice and the full citation on the first page. Copyrights for third-party components of this work must be honored

For all other uses, contact the owner/author(s).

ESEC/FSE '21, August 23-28, 2021, Athens, Greece

(C) 2021 Copyright held by the owner/author(s).

ACM ISBN 978-1-4503-8562-6/21/08.

https://doi.org/10.1145/3468264.3468581 to interact with web applications. For example, users with disabilities may employ Assistive Technology (AT), such as screen readers, speech-based controllers, and switch devices. Since the keyboard is the most universally supported alternative input method operable by people with disabilities [12], most AT generate emulated keystrokes to navigate a web application's User Interface (UI). Unfortunately, keyboard accessibility is one of the most common and prevalent accessibility issues $[6,50,70,71]$. Studies show that as many as $48 \%$ of popular websites lack keyboard accessible mechanisms [66] and $4 \%$ contain accessibility bugs that make the keyboard completely unusable [52]. We call the manifestation of these issues Keyboard Accessibility Failures (KAFs), which are, broadly, failures that prevent the user from interacting with the web app's UI features using the keyboard.

Testing for KAFs is challenging due to the highly complex nature of client-side UIs in modern web applications. These UIs provide responsive and dynamic interfaces that are highly mutable and contain content that can be dynamically created and binded with JavaScript. This poses several challenges: First, the static HTML source code may not resemble the Document Object Model (DOM) with which end-users actually interact, making traditional static analysis on HTML source code inadequate. Second, the keyboard interaction governed by the event-driven execution environment may be constantly changing as the web application enters different UI states. This makes it necessary to identify a web application's possible behaviors, beyond simply a one-time "snapshot." Finally, the JavaScript code responsible for event handlers and their interactions can be difficult to analyze using static analysis, which makes it difficult to statically model keyboard handling in a UI. Together, these challenges can make it very difficult to adequately test for keyboard accessibility issues.

Existing techniques for identifying keyboard inaccessibility issues have limitations in their usage and applicability. For example, Fona [66] statically analyzes a web page's DOM content to identify KAFs. However, it only analyzes specific attributes of DOM elements and does not completely capture all the different ways $\mathrm{KAFs}$ can be introduced into a web page. Other approaches, such as Pyccuracy [68] and aria-check [67] allow developers to write or use scenario based tests to check keyboard accessibility requirements. However, these technique require developers to employ manually generated test cases for each of their pages and anticipate the problems that may occur. As a result, keyboard accessibility testing largely remains a manual effort that requires visual inspections of a web page [10,26], which makes it a time consuming [69], costly, and error-prone [51] process.

In this paper we present our novel approach for automatically detecting and localizing KAFs in web pages. To achieve this, our approach combines both static and dynamic analysis based techniques 

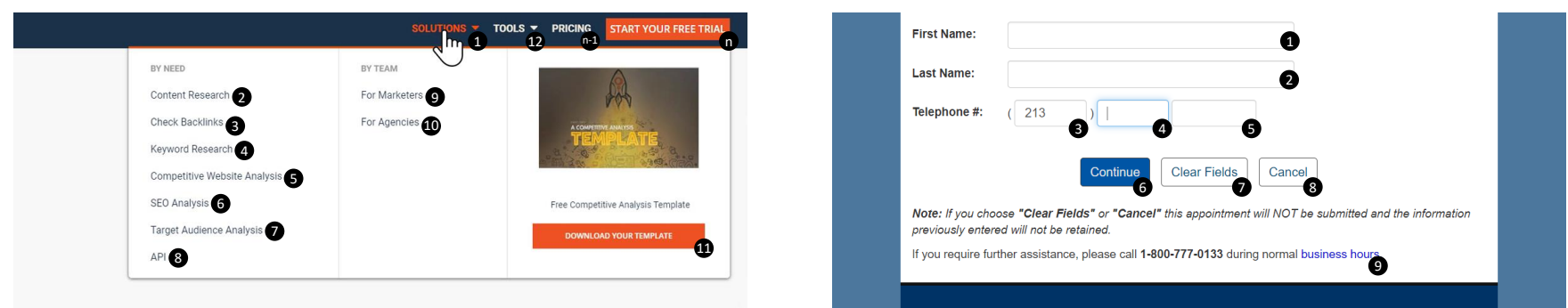

Figure 1: Real-world examples of KAFs (Alexa - left; California DMV - right)

to both address the complications caused by complex modern web app UIs and more completely identify behaviors that can cause KAFs. Our approach defines a dynamic web crawling technique that systematically builds a model representing the possible keyboard based navigation through a web page's UI elements, including those induced by JavaScript based behaviors. Then our approach analyzes this model to identify when the present or missing navigation flows will lead to a KAF. Our evaluation of the technique showed that it is very accurate, in terms of precision and recall, in detecting KAFs and identifying the HTML code responsible for the observed failures. The key contributions of this paper are (1) The first defined keyboard-based navigational model for a web page, (2) The first formalization of keyboard accessibility issues in terms of graph-based properties, (3) A fully automated detection and localization technique that outperforms state-of-the-art and practice on real-world web applications, and (4) A study on a set of real-world web pages that shows that our approach is accurate and efficient.

\section{BACKGROUND}

Keyboard usage is one of the foundational requirements for web accessibility and it is reflected in Guideline 2.1 of the W3C Web Accessibility Initiative (WAI)'s Web Content Accessibility Guideline (WCAG) 2.1 [21]. These guidelines require web UIs to be usable by the many users that operate the computer solely with a keyboardbased input device. Keyboard-based users utilize a set of standard keyboard commands to navigate to different items of interest in web applications and carry out a desired action [20].

Keyboard-based users and point-and-click (PNC) based users interact with a web page's UI elements in a very different manner. In the PNC modality, users move a cursor using a pointing device (e.g., mouse) and press buttons to activate a UI element underneath the cursor. In the keyboard modality, users must press keyboard buttons, such as $\mathrm{Tab}$ and Shift $+\mathrm{Tab}$, to move the browser's focus to a UI element that the user wants to interact with. Unlike the PNC modality, moving between UI elements with a keyboard happens sequentially; meaning that if a user is currently on element $n$ and wants to interact with element $n+i$, then the user must press the Tab $i$ times until focus is on the desired element. The user may also press Shift + Tab to move backwards through the elements. The ordering of the elements is determined by the browser based on the structure of the DOM, but may be overridden by a developer using JavaScript or HTML attributes, such as tabindex. In this paper, we call the ordering of the elements in this sequence the keyboard navigation flow of the web page's UI. Once an element has focus, a keyboard user can carry out actions on it by pressing other keyboard buttons. For example, when the focus is moved onto a group of elements, such as menu lists or radio-buttons, the arrow keys $\uparrow \rightarrow \downarrow \leftarrow \rightarrow$ can be used to move between the elements of the group. The Space or Enter keys are used to manipulate the element currently in focus and the Esc key is typically used to exit a user prompt or dialog [26]. All web browsers are required by the W3C's User Agent Accessibility Guidelines [23] to support this standard set of keyboard strokes through their keyboard API.

Although Keyboard Accessibility Failures (KAFs) can refer to a wide range of keyboard related accessibility issues, in this paper, we use the term to specifically refer to two common types of KAFs that impact the keyboard navigation flow of a web page's UI. These are: (1) Inaccessible Functionalities (IAFs) and (2) Keyboard Traps (KTFs), which are formally defined under WCAG Success Criteria (SC) sections 2.1.1 and 2.1.2. We explain these two types in detail below.

\subsection{Inaccessible Functionalities}

SC 2.1.1 requires all functionality of a web page to be available via the keyboard interface. The term Inaccessible Functionality (IAF) is used to describe failures of a web page UI to conform with this guideline. This type of KAF occurs when an interactive element is not included in the keyboard navigation flow of the UI or an element in the navigation flow does not have a keyboard event handler. This means that a keyboard-based user is either unable to use the standard keyboard navigation keys to put focus on one or more elements in the page's UI or that the element is not actionable and the user is unable to activate the UI element's functionality. There are many root causes of this type of failure, which we discuss in depth in Section 5.4.2, but most relate to custom control elements that do not have event handlers properly defined.

An example of inaccessible functionality occurs in the header navigation menu of the Alexa website, which we found in our Evaluation and is shown in Figure 1. In this subject the "solutions" $(\mathbf{(})$ and "tools" (12) menu items expand sub-menu items when the mouse cursor is hovered over them. These menu items are implemented with $<$ div $>$ elements that have the :hover CSS pseudo-class [13] defined in the web page's static CSS declarations to make them interactable. Since the expansion of the sub-menus are only triggered with mouse hover, the contained links (2 to (1)) are not accessible to keyboard-based users, resulting in a situation where none of the sub-menu items can be seen by a keyboard-based user.

\subsection{Keyboard Traps}

SC 2.1.2 requires that if keyboard focus can be moved to a UI element of the page using the keyboard interface, then focus must also be able to move away from that element using only the keyboard 
interface. The term Keyboard Trap (KTF) is used to describe failures of a web page UI to conform with this guideline. This type of KAF occurs when focus becomes "stuck" on a sequence of one or more interactive elements and the user is unable to interact further with other parts of the web page via the keyboard. As with the first type of KAF, there are many possible root causes for this kind of KAF, which we discuss in depth in Section 5.4.2, but the most common is the use of third-party widget plugins that once receiving focus, prevented the user from returning to content outside of the plug-in via the keyboard.

Figure 1 shows an example of a KTF that we found in one of our Evaluation subjects, the California DMV's appointment registration page [29]. This trap was caused by JavaScript that auto advanced the keyboard cursor when the user typed part of a phone number. When three numbers were entered into the area code input field (3), developer-defined JavaScript code automatically advanced the cursor to put focus on the next input field (4). Consequently, a keyboard user was not be able to make a correction to the area code by moving backwards, since on each attempt to shift focus to the area code input box would cause the JavaScript to again advance focus to the next input field (4). In fact, any interactive element prior to the trap (e.g.,(1) to (3) was no longer accessible to the keyboard user after entering an area code.

\section{WEB APPLICATION MODELING}

To perform the detection and localization of KAFs, our approach first takes a Page Under Test (PUT) as input and builds models of the ways users can interact with it via both the keyboard and PNC. This is shown in the left half of Figure 2. The Keyboard Navigation Flow Graph (KNFG) models the different ways a keyboard user can interact with a PUT. To the best of our knowledge, we are the first to define such a model. The Point-Click Navigation Flow Graph (PCNFG) represents the ways a PNC user can interact with a web page's UI and is similar to existing representations, such as the state flow graph [57].

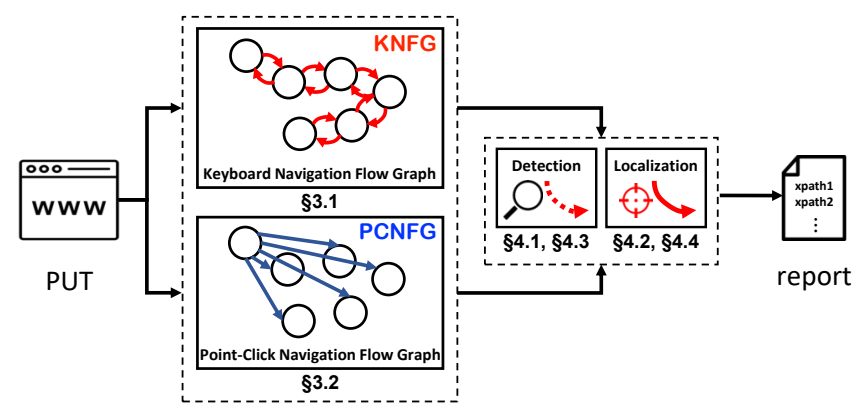

Figure 2: An overview of our approach

\subsection{Modeling Keyboard Navigation Flow}

KNFG Formal Definition. To model keyboard navigation flow, we define a new abstraction, the Keyboard Navigation Flow Graph (KNFG). A PUT's keyboard navigation flow is represented by a set of KNFGs. Each KNFG represents the ways a web page's UI can be navigated from the perspective of a keyboard user. Formally, we represent a KNFG as a tuple $\left\langle V, V_{s}, v_{0}, \Phi_{K}, E\right\rangle$, where $V$ is the set of all nodes in the graph and each $v \in V$ corresponds to a unique HTML element in the PUT, $V_{s} \subseteq V$ represents a state of the PUT, which we define as the set of HTML elements that are visible in the PUT, $v_{0} \in V_{s}$ is the entry node of the graph (i.e., the first HTML element that keyboard-based users can interact with when the PUT renders in the browser); $\Phi_{K}$ is the set of standard keyboard-based actions that may cause a focus transition between nodes in the graph; and $E$ is the set of directed edges that represent the transitions that can occur in the PUT.

KNFG Nodes. The node set of a KNFG, $V$, contains a node for each HTML element in the PUT. Our approach identifies the nodes by rendering the PUT in a browser and then analyzing the PUT's DOM to identify each unique HTML element. Each node is uniquely identified by its XPath in the DOM. Our approach groups syntactically linked nodes such as a $<$ label $>$ and its bounded form element and elements wrapped within other inline control elements, since these nodes are intended to represent a single functionality. A node $v_{0} \in V$ represents the entry node of the graph and is the HTML element in the page that is the starting point for keyboard-based navigation of the page. A PUT's initial $v_{0}$ node can be identified by determining the XPath of the element that initially has focus after the page renders in the browser.

The inclusion of state $\left(V_{s}\right)$ in the KNFG enables our approach to model modern web applications where new HTML elements may be enabled or added to a PUT. We define $V_{s}$ as the subset of all nodes in the PUT that are visible in the browser. Although related work has proposed many definition of state for web pages, we use this because it is well-suited for our problem domain, since only visible elements can be interacted with via the keyboard and any change in the set of visible elements may introduce a new navigation flow. To illustrate, consider Figure 3, which shows a hidden $<$ div $>$ component that is not displayed until the user activates the "Forgot Name" link - its event handling triggers and expands the hidden $<$ div $>$, displaying the encompassed three "Phone Lookup" text fields. Our approach would consider 1, 2, and $\mathbf{3}$ to be distinct states, since the additional displayed links will define a new navigation flow through the PUT. A challenge in identifying the HTML elements that comprise $V_{S}$ is that due to the complex interactions between HTML and CSS attributes, there is no canonical way to determine if a given node is visible. Therefore, we compiled a set of heuristics that our approach uses to determine if an element is visible. These are (1) non-disabled elements that do not exhibit a final computed DOM layout style of type="hidden", visibility: hidden, display: none, or inherit their ancestor's rendered hidden properties; (2) elements that are not rendered with a height or width of zero pixels; or (3) excluded from the PUT's visual flow (e.g., elements inside another tab-menu, or inside containers that are collapsed whose contents aren't shown). All of these heuristics can be identified by analyzing the attributes of the elements in the DOM of the PUT when it has been rendered in the browser.

KNFG Edges. The edge set $E$ of a KNFG represents the keyboard navigation flow possible as a result of keyboard-based actions. A keyboard user can manipulate a page's interactive elements using a standard set of actions, which we represent with $\Phi_{K}$. This set of actions includes all standard keyboard commands used to navigate a web application's UI as defined by W3C [24] and web accessibility testing communities $[10,19,26]$. $\Phi$ 's operations can be broken 

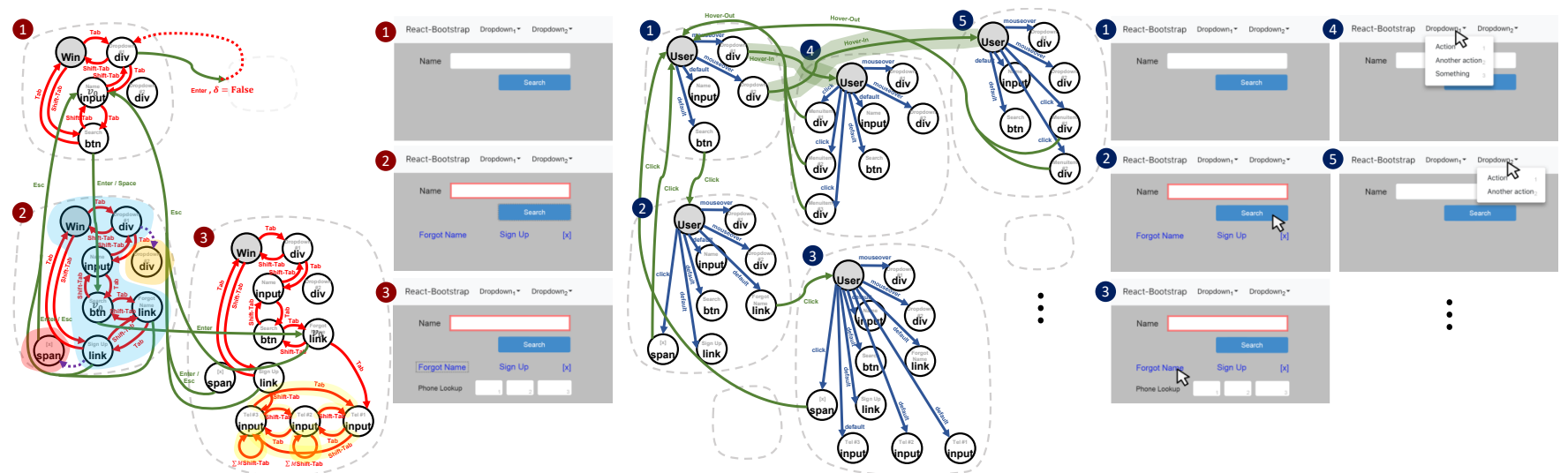

Figure 3: A simplified version of the $\mathrm{KNFG}^{*}$ (left) and PCNFG* (right) with their corresponding UI states (omitted some noninteractive nodes and self-loop edges incurred by $\uparrow, \downarrow, \leftarrow, \rightarrow$, , Enter, Space, or Esc)

into four categories: navigation $(\mathrm{Tab}$, Shift $+\mathrm{Tab})$, selection $(\uparrow$, $\downarrow, \leftarrow, \rightarrow$ ), actuation (Enter, Space) and dismissal (Esc). Our approach does not model keyboard shortcuts or scrolling keys such as PgUp, PgDn, Home, and End since they only change how contents are displayed (or move the keyboard cursor within a text input-based control) and serve no interactive purposes. To trigger navigation behaviors that require text input, we also include two actions that represents the entry of text. The first is $\Sigma$ and represents the entry of a random length string of alphanumeric characters. The second is $\Sigma_{M}$ and represents the entry of a maximum length string into the text input, where maximum length is determined by the maxlength attribute of the text input, if defined. Both text entry actions are followed by the Tab, Shift $+\mathrm{Tab}$, or no action. This simulates the common user behavior of entering input or interacting with a control, and then moving to either the next or prior element in a page. In the text, we will denote these sequences by showing both relevant symbols as the action, even though they can be represented as a single compound action (e.g., entering max length text and then pressing tab would be represented as $\Sigma_{M}$ Tab)

KNFG Construction. At a high level, the edges in the KNFG are identified by iteratively exploring the page using only keyboardbased actions (i.e., $\Phi_{K}$ ) until no new navigation information is found (i.e., the graph has reached a fixed point). The first iteration of this process begins by interacting with each node $v \in V_{s}$. For a given $v$, our approach first sets the browser's focus on $v$ and then executes every action in $\Phi_{K}$ on $v$. After each action, our approach analyzes the page to determine the focus transition that occurred. If an action $\phi$ causes a new state to be created, then the new state is added to a worklist of states that will be explored in subsequent iterations of the edge building process. If the action causes the page to attempt to navigate to a different page, our approach intercepts this event and prevents it from happening. Our approach also includes two edges that link the last element in a page's tab order to the first (i.e., $v_{n} \rightarrow v_{0}$ and $\left.v_{n} \leftarrow v_{0}\right)$. This is done in order to exclude browser controls, which are accessible via Tab, from the KNFG. An edge can be either an intra or inter state edge. We describe the details of handling each kind of edge below.

Intra-state edges describe the navigation flow in a page as a result of an action on a node when there is no new page state created. After triggering an action $\phi \in \Phi_{K}$ on a node $v_{i}$, our approach detects the focus change from $v_{i}$ to $v_{i+1}$ and creates an edge in the graph $\left\langle v_{i}, v_{i+1}, \phi, \delta, V_{s}\right\rangle$, indicating that the browser focus could shift from a source node $v_{i}$ to a target node $v_{i+1}$ by pressing keystroke $\phi$ while $v_{i}$ is in focus. Our approach detects a change in focus by querying the browser to identify the element currently receiving focus in the PUT. If an action does not trigger a focus-shift (e.g., executing Space to tick a checkbox), a self edge is created. Our edge creation mechanism can capture a shift (or no shift) in focus that is caused by JavaScript event handlers since it simply observes the response of the page to the keyboard action, which would include any JavaScript actions. Lastly, if $\phi$ causes any sort of change in the DOM's attributes' values, then the $\delta$ flag is set to True, otherwise it is set to False. Using our running example, an intra-state edge exists between the "Name" input-field to the "Search" button since focus can transition between the two elements by pressing the Tab and no new visible HTML elements appear (i.e., there is no new state created). This edge and other intra-state edges for our example are shown in red in Figure 3.

Inter-state edges describe the navigation flow in a page when an action on a node leads to the creation of a new UI state. When our approach detects a new state, it creates an edge in the same way that an intra-state edge would be created with the exception of the target node. In this scenario, the target node is in the new state $\left(V_{s}^{\prime}\right)$ and is the node $v_{0} \in V_{s}^{\prime}$ to which focus is transferred to by the browser. An example of an inter-state edge can be seen in our running example where pressing Esc on the "Forgot Name" and "Sign Up" links in state 2 (when the < div> component containing these links is active) will dismiss the $<$ div $>$ component and return the navigation to state 1 . This edge and other inter-state edges are represented as green edges in Figure 3.

The KNFG construction is sound under the assumption that the keyboard navigation is deterministic (i.e., executing $\phi$ on every node would always transition the focus to the same node and a node has exactly one predecessor/successor through an intra-state edge). In the cases where non-determinism did occur (an element could transition to different elements), we observed such cases were always accompanied by some kind of visual state change, which would be captured in the KNFG building process. The edge construction is complete with respect to the W3C default set of standardized keyboard navigation keys. If a web page were to use 
non-standard keys for interaction, our approach would not explore these actions. However, it is important to note that these cases are not common in practice and our $\Phi_{K}$ could be easily redefined to handle other key sets. The node identification is complete, since all nodes in a page can be identified by examining the DOM of the page at runtime. The only exception to this is for web sites that embed objects, such as legacy Flash, Silverlight, Java Applets, or third-party plugins, neither of which are modeled in the KNFG. However, note that support for these frameworks is currently being phased out by major browsers [8].

\subsection{Modeling Point and Click Navigation}

The navigation and interactions available to a PNC user in a PUT are represented by a set of Point-Click Navigation Flow Graphs (PCNFGs). Each PCNFG represents the ways a web page's UI can be interacted with from the perspective of a PNC user for a particular state of the PUT's UI. Formally, we represent a PCNFG as a tuple $\left\langle V, V_{s}, v_{u s e r}, \Phi_{M}, E\right\rangle$, where $V$ is the set of all nodes in the graph, $V_{s} \subseteq V$ represents the state, which is defined by the set of nodes that are visible in the PUT, $v_{\text {user }} \in V$ is the initial node; $\Phi_{M}$ is the set of mouse event types; and $E$ is the set of edges that represent the mouse navigation control flow.

Information about the nodes in the PCNFG is defined similar to that of the KNFG. Specifically, the node set $(V)$ and state $\left(V_{s}\right)$ of the PCNFG are defined and identified in the same way as those for the KNFG. The one exception to this is the definition of the entry node $v_{\text {user }}$ of the graph. Unlike the case of keyboard-based navigation, there is not a single node in the PUT that represents the starting point for PNC based navigation. Therefore, we define a special node $v_{\text {user }}$ that conceptually represents the entry to the PUT and connects to all of the nodes that a PNC user can interact with in the PUT.

A PNC based user can manipulate a page's interactive elements using a set of actions, which we represent with $\Phi_{M}$. This set includes all mouse event types that implement the MouseEvent interface [22], such as mousedown, mouseup, click, mouseover, mouseenter, mouseleave, or mouseout. The edges in the PCNFG are defined using a standard iterative crawling exploration. For example, similar to the construction of the state flow graph originally proposed by Crawljax [57]. In our approach, the target elements that will have events triggered on them are represented by $V_{S}$ and the events to be triggered on them are represented by $\Phi_{M}$. We omit further details of this exploration due to space constraints and since it is not a contribution of our approach. An example UI and PCNFG are shown in Figure 3.

\section{DETECTION AND LOCALIZATION OF KAFS}

As shown in Figure 2, once our approach has generated the KNFGs and PCNFGs for the PUT, it detects and localizes KAFs by analyzing and comparing the two graphs.

\subsection{Detection of Inaccessible Functionalities}

An IAF occurs when functionality available to a point-and-click (PNC) user is not available to a keyboard user. At a high level, our approach identifies all of the interactive HTML elements available to a PNC user by analyzing the PCNFG and then, by analyzing the KNFG, checks that the same elements can be navigated to and activated by a keyboard user.

Our approach to detect IAFs is shown in Algorithm 1. The inputs to this algorithm are the set of PCNFGs and KNFGs that were previously constructed. We denote these sets as $\mathrm{PCNFG}^{*}$ and $\mathrm{KNFG}^{*}$, respectively. First, the algorithm identifies the functionality (i.e., interactive elements) available to a PNC based user (line 2). This is done by calculating $V_{P C}$ the set of nodes in the $\mathrm{PCNFG}^{*}$ that are reachable from $v_{\text {user }}$ (i.e., the entry node) of the initial state of the $\mathrm{PCNFG}^{*}$. Next the approach iterates over each node $v_{p c} \in V_{P C}$ and checks if it is also accessible to a keyboard user (lines 3-8). For each $v_{p c}$, the approach finds the corresponding node $v_{k}$ in the KNFG* (line 4). Note that for a given state, both the PCNFG and KNFG have the same $V$ set, and therefore there will always be a corresponding node if the corresponding KNFG state exists.

Non-reachable IAFs. The first accessibility check (line 5) determines if $v_{k}$ is reachable from $v_{0}$ of the KNFG*. If it is not, then this implies a keyboard user cannot navigate to $v_{k}$ (i.e., $v_{k}$ cannot receive keyboard focus) using the keyboard navigation flow defined by the PUT and $v_{k}$ is considered to be inaccessible and added to $V_{I F}$, the set of elements inaccessible due to flow problems (line 6).

Non-actionable IAFs. The second accessibility check (line 7) determines if $v_{k}$ is actionable. A naive way to detect this would be to simply examine the DOM and determine if $v_{k}$ has either an explicit or implicit keyboard event handler associated with it. However, a keyboard event handler may be assigned through complex event delegation, which may not be visible via DOM inspection, or $v_{k}$ may handle some key presses (e.g., $\mathrm{Tab}$ and Shift $+\mathrm{Tab}$ ) that simply change the browser focus without actually activating the element. Therefore, to determine if $v_{k}$ is actionable it is necessary to check in the KNFG whether it has any outgoing edges that modify the DOM state (i.e., $\delta$ is set to True). The presence of such an edge indicates that there exists some keyboard action on the element that can be triggered and do something other than transfer focus to another element. Note that WCAG only requires actionable as an accessibility criteria when the element can be triggered by the mouse. This is accounted for in our approach since $v_{k}$ is the corresponding node of a node already in the PCNFG, and, by definition, a node is only in the PCNFG if it has an associated mouse event handler.

Once all nodes in $V_{P C}$ have been analyzed, the detection returns True/False based on whether any nodes have been identified as inaccessible (line 9). This algorithm can be optimized for page-level detection by returning True the first time any inaccessible element is identified (i.e., at lines 6 and 8).

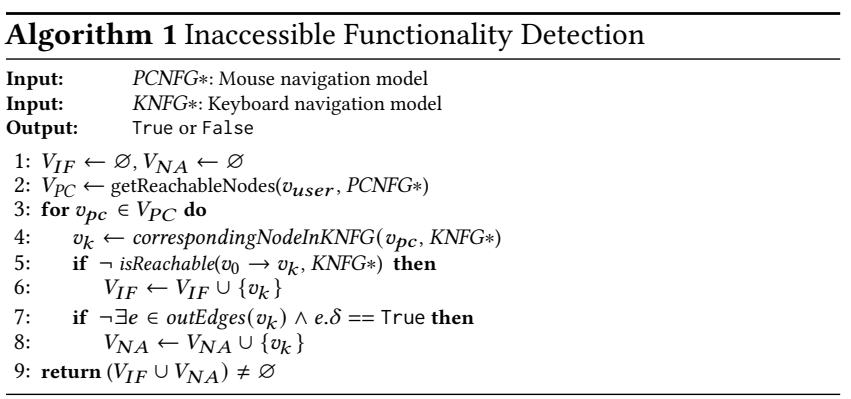


In the $\mathrm{KNFG}^{*}$ from our running example (Figure 3), the algorithm identifies that in state (1), $v_{\text {dropdown } 2}$ must be accessible via the keyboard because it is reachable in the PCNFG. However, in the KNFG, it is unreachable from $v_{0}$ via flow edges (red edges), thus putting this node in $V_{I F}$. Similarly for $v_{\text {dropdown } 1}$, the detection finds it is reachable from $v_{0}$ but finds there is no outgoing edge with $\delta=$ True (such an edge would exist if its Enter edge could have explored state (4). Therefore this node is put into $V_{N A}$.

\subsection{Localization of Inaccessible Functionalities}

When Inaccessible Functionalities occur, it implies that there is either (a) an edge missing from the KNFG that would connect the accessible components to the inaccessible components (i.e., not reachable) or (b) an element is missing a required keyboard handler (i.e., not actionable).

Non-reachable IAFs. To localize the missing edges that cause elements to become unreachable, our approach analyzes the PCNFG* and $\mathrm{KNFG}^{*}$ and assigns a suspiciousness score to various edges that could represent the most likely connection point. At a high-level, the intuition of our approach is to split the localization into two parts. The first part tries to identify the most likely source node of the missing edge and the second part tries to identify the most likely target node of the missing edge. Our approach computes suspiciousness scores for each of these two node sets and then presents a ranking of the edges based on the combined suspiciousness of the source and target nodes of the edge. The algorithm for computing suspiciousness of each edge is shown in Algorithm 2. The inputs to this algorithm are $\mathrm{PCNFG}^{*}$ and $\mathrm{KNFG}^{*}$, as defined above. The algorithm iterates over each state's PCNFG and checks to see if the state of the PCNFG exists in the KNFG*. Since both types of graphs have the same $V$ set and define their states in the same manner, this check only needs to determine if the $V_{s}$ for the PCNFG exists in the $\mathrm{KNFG}^{*}$.

If the state is present in the $\mathrm{KNFG}^{*}$, the approach examines the state to determine if it contains nodes that are inaccessible (i.e., unreachable). Lines 5-8 compute the set of nodes that are inaccessible for the keyboard user $\left(V_{K A F}\right)$, using a process similar to that defined in Algorithm 1. The approach then iterates over each node $v_{k a f} \in V_{K A F}$ in order to determine the most likely source and target nodes for the missing edge that caused $v_{k a f}$. The possible source nodes are represented as $V_{S}$, which was already computed as the set of nodes reachable in the KNFG from $v_{0}$ at line 7 . Since these nodes are reachable from the entry point, one of them will be the node that represents the source node of the missing edge. Conversely, $V_{T}$ represents the set of possible missing edge target nodes. The approach computes $V_{T}$ by finding all of the nodes that can reach to $v_{k a f}$. One might assume that the missing edge target node would always be $v_{k a f}$, however it is possible that $v_{k a f}$ may be part of a larger component, which is also inaccessible. In this scenario, these other nodes should also be considered as possible targets. For nodes in $V_{T}$, line 16 computes their suspiciousness score. The intuition of this score is that nodes in $V_{T}$ represent a subgraph of the KNFG and nodes higher in the topological ordering of this subgraph are most likely the correct target node, since they can themselves connect to the most nodes in the subgraph. Therefore, rank assigns these nodes a higher suspiciousness. Specifically, the DOM based rank of each of the nodes in $V_{T}$ is used to assign suspiciousness. A suspiciousness score is also computed for nodes in $V_{S}$. In lines 13-14, our approach iterates over each of the nodes in $V_{S}$ and assigns a suspicousness score to each. The basic idea is that nodes in $V_{S}$ are considered more likely to be the source node if they are spatially "close" to nodes in $V_{T}$. We define "close" as the DOM tree based distance between the two nodes, which is calculated by the number of edges that must be traversed in the DOM tree for one node to reach another. This definition of closeness represents the design practice that keyboard navigation generally transitions between nodes that have some kind of locality. The approach then takes the Cartesian product of $V_{S}$ and $V_{T}$ and assigns the suspiciousness score of the edge $\left(v_{s}, v_{t}\right)$ based on the sum of the suspiciousness scores of $v_{s}$ and $v_{t}$. The approach resets the node scores for $V_{S}$ and $V_{T}$ every time it processes a $v_{k a f}$ so the scores always reflect the suspiciousness with respect to the inaccessible components. Note that the final scores of the edges in $E$ are cumulative across all states (lines 18-19).

In our running example (Figure 3), the localization algorithm identifies that in state 2 of the $\mathrm{KNFG}^{*}, v_{\text {dropdown } 2}$ and $v_{[x]}$ must be accessible via the keyboard, but since neither nodes are reachable from $v_{0}$ in that state, both are in the set $V_{K A F}$. When identifying the edges responsible to connect these nodes, the algorithm first identifies $V_{S}$ as the blue cluster reachable from $v_{0}$ and $V_{T}$ as the orange cluster where $v_{\text {dropdown } 2}$ resides. The algorithm then identifies the purple dotted edge as the candidate faulty edge with the highest suspiciousness score due to their proximity in the DOM. This concept also applies to $v_{[x]}$ in its red cluster.

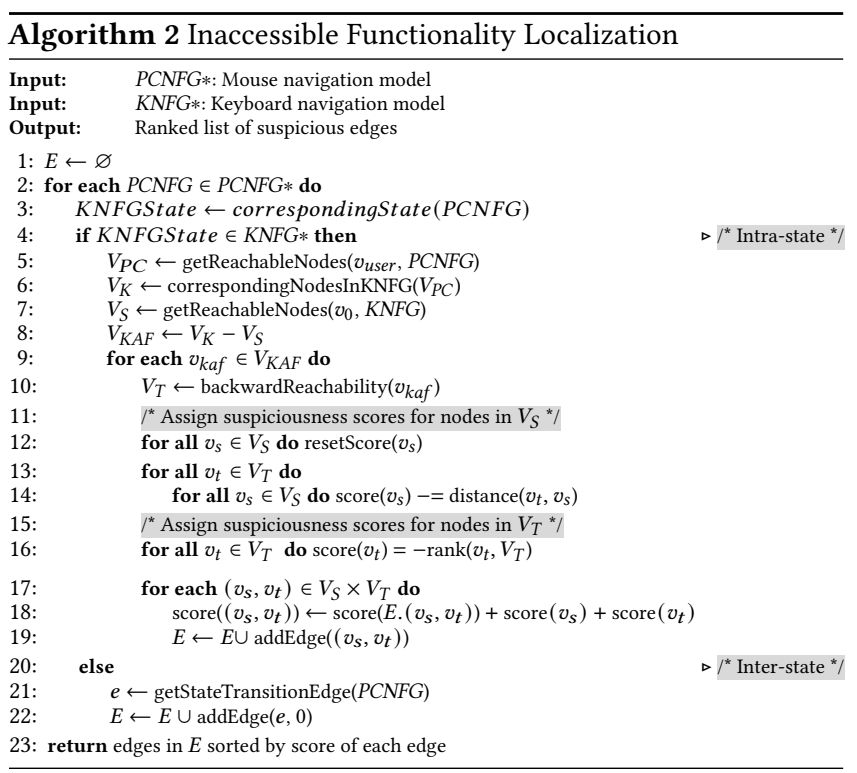

If the current PCNFG state does not match a state in the KNFG, then the identification of the missing edge is simplified (line 4 and 21-22). Because of the way our approach constructs the $\mathrm{KNFG}^{*}$, a new state in the $\mathrm{KNFG}^{*}$ is only defined if there exists some action (an edge) that during the crawling of the current KNFG state transitions the state's $V_{s}$ to $V_{s}^{\prime}$. Thus, if a state exists in the $\mathrm{PCNFG}^{*}$ and not in the KNFG* ${ }^{*}$, it indicates (1) there exists some missing keyboard 
navigational transition leading to the $V^{\prime}$ state, and (2) the state transition edge to $V^{\prime}$ state in the PCNFG* is the edge most likely to be faulty. Line 22 classifies the missing state transition edge with the highest possible suspiciousness score of 0 . For example, in the $\mathrm{PCNFG}^{*}$ of our running example, state $\mathbf{4}$ and state $\mathbf{5}$ are explored via mouse-hovering Dropdown1 and Dropdown2. The algorithm identifies the inter-state edge (highlighted in green) that transition from these nodes to $\mathbf{4}$ and $\mathbf{5}$ as suspicious edges because these states are not in the KNFG*.

Non-actionable IAFs. The localization of elements that are not actionable is exactly the set of elements identified as $V_{N A}$ in Algorithm 1 and no further localization is needed for those elements.

\subsection{Detection of Keyboard Traps}

To understand the intuition of our detection approach for Keyboard Traps, first recall that Keyboard Traps cause keyboard-based users to become "stuck" in a sequence of one or more nodes. The navigation flows that would cause this to occur will be identifiable as cycles in the KNFG. This represents a situation where once a keyboard user has entered the cycle with either Tab or Shift $+\mathrm{Tab}$, they will be unable to navigate away from the elements in the cycle using the keyboard.

The approach to detect Keyboard Traps is shown in Algorithm 3. The algorithm begins by iterating over each KNFG in the KNFG ${ }^{*}$ (Line 1). For each KNFG, the approach extracts two edge-induced subgraphs, one based on edges labeled with the Tab action and the other one based on edges labeled with the Shift + Tab. The two subgraphs are computed by iterating through the edge set $E$ of the KNFG and retaining the subset of edges where $\phi=$ Shift + Tab or $\mathrm{Tab}$. These two subgraphs represent the two ways keyboardbased users are able to navigate among elements and that would be affected by a cycle. Using the edge induced subgraphs enables the approach to avoid false-positives that would be caused by cycles defined by the forward-backward edges created for each node due to the $\mathrm{Tab}$ and $\mathrm{Shift}+\mathrm{Tab}$ navigation and by edges whose actions may not cause a focus shift, such as $\uparrow, \downarrow, \leftarrow, \rightarrow$, Enter, or Space. The approach then iterates over the two subgraphs (Line 4). The approach first removes the specially created edges that link the last element in a page's tab order and the first element (i.e., $v_{n} \leftrightarrow v_{0}$ ) (Line 5). The reason for this removal is that this specially added edge creates a cycle in the KNFG to enable users to cycle from the last node in the tab order to the first, but does not represent a Keyboard Trap. After the edge removal, our approach analyzes the subgraph to determine if it contains a cycle, in which case the approach has identified that a KTF exists in the KNFG (Line 6).

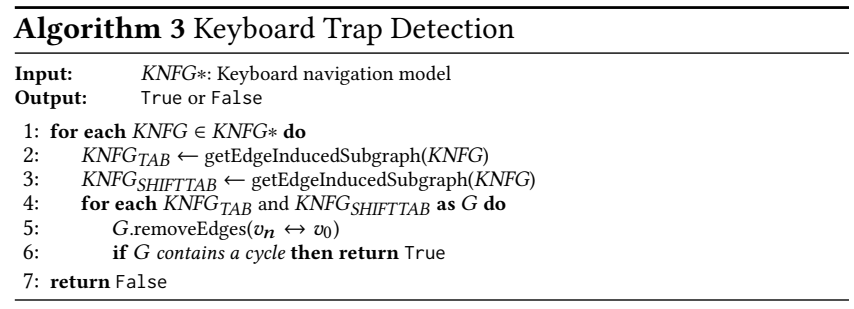

In state 3 of the $\mathrm{KNFG}^{*}$ from our running example, the four cycles highlighted in yellow would be detected. Of which, the two cycles of size 1 formed by edge $\left\langle v_{t e l 2}, v_{t e l}, \Sigma_{M}[\right.$ Shift $\left.+\mathrm{Tab}], V_{s}\right\rangle$ and $\left\langle v_{t e l 3}, v_{t e l 3}, \Sigma_{M}[\mathrm{Shift}+\mathrm{Tab}], V_{s}\right\rangle$ are results of the auto-tabbing mechanism. The larger two cycles of size 3 formed by edges $\left\langle v_{t e l 1}, v_{t e l 2}\right.$, $\left.\mathrm{Tab}, V_{s}\right\rangle,\left\langle v_{t e l 2}, v_{t e l 3}, \mathrm{Tab}, V_{s}\right\rangle,\left\langle v_{t e l 3}, v_{t e l 1}, \mathrm{Tab}, V_{s}\right\rangle$ and $\left\langle v_{t e l 1}, v_{t e l 3}\right.$, Shift $\left.+\mathrm{Tab}, V_{s}\right\rangle,\left\langle v_{t e l 3}, v_{t e l 2}, \mathrm{Shift}+\mathrm{Tab}, V_{s}\right\rangle,\left\langle v_{t e l 2}, v_{t e l 1}, \mathrm{Shift}+\mathrm{Tab}, V_{s}\right\rangle$ are the result of the mechanism that prevents the user from leaving the three telephone boxes unless entries have been filled.

\subsection{Localization of Keyboard Traps}

When a KTF occurs it indicates that an edge has been created that should not be present in the navigation. The key insight is that a KTF is caused by an edge that already exists but whose transition to the next node in the navigation flow is incorrect. Therefore our analysis tries to identify which edge in the KNFG is mostly likely to be incorrect. Since there is not a definitive way of identifying this edge, we employ a suspiciousness score based approach that encodes heuristics that we found useful in identifying the likely faulty edge.

The approach for computing suspiciousness of the edges is shown in Algorithm 4. Lines 2-6 employ the same preparatory steps used in the detection algorithm (Algorithm 3). Namely, the approach iterates over each KNFG of the PUT, identifies the two edge induced subgraphs in the KNFG, and then removes the edges between $v_{n}$ and $v_{0}$ of each subgraph. Both heuristics employed by our approach focus on the nodes and edges that are part of a cycle (i.e., the detected KTF). Therefore, the next steps of the algorithm (lines 7-9) identify and extract the connected components in the graph. Our approach does this by using Tarjan's bridge-finding algorithm [27] to find and remove all bridges (i.e., edges that disconnect a component when removed) in $G$ (lines 7-8). This leaves only connected components in $G$. Since this set of components may include components of size one with no self-loops, the approach removes these nodes (line 9.) These nodes can be safely removed from consideration since, by definition, they cannot be part of a trap (i.e., they do not contain a self loop and are not part of a larger cycle.)

The next part of the algorithm (lines 10-19) applies our approach's localization heuristics. Our approach employs two heuristics for localization. The first heuristic is that edges in a connected component are more likely to be a faulty edge than those not in the component. This heuristic is reasonable since typically one of the edges in the component's cycle should actually be directed to a node not in the cycle (i.e., breaking the cycle), and the failure to do so is the cause of the KTF. This heuristic is implemented in lines 11-13, which iterate over the edges in the component and increase the suspiciousness score of each of them. The second heuristic focuses on identifying the edge in the cycle that is most likely to represent the back-edge with respect to the page's navigation flow. The key insight to doing this is that the default tab navigation rendered by the browser is determined by a depth first, pre-order traversal of the DOM tree [11]. In most cases, unless scripting was used to alter the default tab sequence, the DOM rank determines the keyboard navigation flow. In lines 14-17, our approach leverages this information to identify the most suspicious edge within the cycle. Specifically, for the Tab induced subgraph, this edge is the one that flows from the lowest (min) DOM ranked element to the highest 
(max) DOM ranked element (line 15). For the Shift $+\mathrm{Tab}$ induced subgraph, since this goes in the reverse direction, it is the edge that flows from the highest $(\max )$ DOM ranked node to the lowest (min) DOM ranked node (line 17). Note that such back-edge may not exist if the default sequence of tab navigation is violated. In such extremely uncommon cases, the heuristic would still work, but not as well (i.e., it would lower the faulty element's ranking). The back-edge identified by this heuristic has its suspiciousness score increased (line 19). These heuristics are applied for each KNFG and once all of them have been analyzed, the approach returns a list of all edges ranked in order of their score, highest to lowest (line 20).

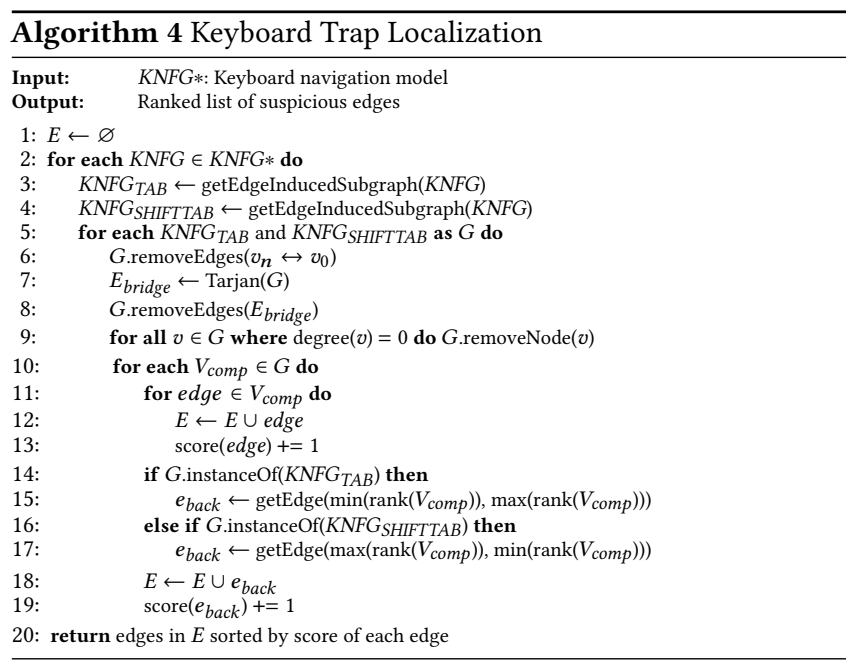

In state 3 of the $\mathrm{KNFG}^{*}$ from our running example, the cycle formed across $v_{t e l 1}, v_{t e l 2}$, and $v_{t e l 3}$ that prevents the user from leaving the three telephone boxes consists of three edges in either the $\mathrm{Tab}$ and Shift $\mathrm{Tab}$ direction. The order of appearance of these three text fields in the DOM sequence would make the edge $\left\langle v_{t e l 3}, v_{t e l 1}, \mathrm{Tab}, V_{s}\right\rangle$ and $\left\langle v_{t e l 1}, v_{t e l 3}\right.$, Shift $\left.+\mathrm{Tab}, V_{s}\right\rangle$ as the respective "back-edge" that is more suspicious.

\section{EVALUATION}

To assess the effectiveness of our approach, we conducted an empirical evaluation that focused on three research questions:

RQ1: What is the accuracy of our technique in detecting KAFs in web applications?

RQ2: If a KAF is detected, what is the quality of the localization results provided by our technique?

RQ3: How much time does our technique need to detect and localize KAFs?

\subsection{Implementation}

We implemented our approach as a Java-based prototype tool, Keyboard Accessibility Failure dEtector (KAFE). The implementation employs a crawler for each subject page's exploration and is fully automated, requiring no manual effort by users. It uses Selenium WebDriver to load, render, and interact with the subject web pages to build the KNFG and PCNFG. All web pages were rendered with a fixed screen resolution of $1920 \times 1080$ pixels on Firefox version 68.0 controlled by Selenium version 3.141.5. During interaction with a subject web page, we used the WebDriver API [35] to send key presses to the page and monitor the switch in focus. We wrote and executed JavaScript-based code to detect changes to the subject page and capture the page's DOM for further analysis. We ran KAFE and all experiments on a single AMD Ryzen Threadripper 2990WX 64-bit machine with 64GB memory and Ubuntu Linux 18.04.4 LTS. The implementation of KAFE and subjects will be made available to the community via the project website [31].

\subsection{Subject Web Pages / Accessibility Tools}

We conducted our evaluation on a set of real-world subject web pages gathered from (1) government and higher education web sites that are required by law to comply with WCAG [2], (2) frequently visited e-commerce web sites, and (3) sites from the Moz Top 500 most visited websites list [17]. We chose subjects from these sources due to their obligation for accessibility by the ADA [43] and their popularity. The authors manually interacted with each web page's UI and the underlying functional components per keyboard accessibility testing techniques [18] and used 40 of those that contained at least one KAF. Overall, our subjects contained 168 IAFs and 28 KTFs. For each KAF, we identified the edge(s) that would either enable access to IAFs or remove cycles causing KTFs, while at the same time remaining consistent with the page's pre-existing navigation flow. To help measure the false-positive detection rate in RQ1, we also included 20 subjects that did not contain any KAFs. The dynamic UI contents in the subjects comprised of modern HTML5 and custom JavaScript widgets, such as sliders, dropdown menus, calendars, and tooltips components. Due to space constraints, we do not list each of the subjects in the paper, but include them as supplementary material in the Appendix. We captured a complete version of each subject web page using an interactive HTTP proxy [16].

For the purpose of evaluating our approach's performance, we compared against a set of state-of-the-art tools from two sources: (1) existing web accessibility testing tools described in research literature, and (2) the Web Accessibility Evaluation Tools List [7] provided by the W3C. From (1), we selected aria-check [67], tabindex-counter (the implementation of the Fona approach [66]), and QualWeb [47] because they detected failures that at least partially overlapped with KAFs. The other tools we found (aChecker [49], EvalAccess [36], Hera-FFX [48], MAUVE [63], Pyccuracy [68]) did not target and were not capable of detecting issues related to keyboard accessibility. From (2), we selected the popular WAVE [25] tool because it had the most complete handling of keyboard accessibility issues of the listed tools. Other tools on the list ignored the impact of scripting and styling that are responsible for most accessibility issues [41], which meant they could not outperform WAVE. For all of these tools, we used the publicly available official implementations via their websites.

\subsection{Procedures and Presentation of Results}

To answer RQ1, we ran KAFE against the subject web pages and measured how accurately it could detect both types of KAFs. In this experiment, we measured the precision and recall of KAFE's detection results and compared this against the accuracy of existing 
Table 1: Detection, localization, and timing results for each evaluated approach.

\begin{tabular}{|c|c|c|c|c|c|c|c|c|c|c|c|}
\hline \multirow[b]{2}{*}{ Tool } & \multicolumn{5}{|c|}{ Inaccessible Functionality Failure } & \multicolumn{5}{|c|}{ Keyboard Trap Failure } & \multirow{2}{*}{$\begin{array}{c}\text { Run time(Minutes) } \\
\text { Average }\end{array}$} \\
\hline & $\operatorname{DEP}(\%)$ & $\operatorname{DER}(\%)$ & $\operatorname{LOR}(\%)$ & \#MEF & \#AEF & DEP(\%) & $\operatorname{DER}(\%)$ & $\operatorname{LOR}(\%)$ & \#MEF & \#AEF & \\
\hline KAFE & 92 & 100 & 94 & 5 & 8.8 & 90 & 100 & 89 & 1 & 4 & 19.22 \\
\hline aria-check & 60 & 100 & $\mathrm{n} / \mathrm{a}$ & $\mathrm{n} / \mathrm{a}$ & $\mathrm{n} / \mathrm{a}$ & 0 & 0 & $\mathrm{n} / \mathrm{a}$ & $\mathrm{n} / \mathrm{a}$ & $\mathrm{n} / \mathrm{a}$ & 0.03 \\
\hline tabindex-counter & 93 & 39 & $\mathrm{n} / \mathrm{a}$ & $\mathrm{n} / \mathrm{a}$ & $\mathrm{n} / \mathrm{a}$ & 0 & 0 & $\mathrm{n} / \mathrm{a}$ & $\mathrm{n} / \mathrm{a}$ & $\mathrm{n} / \mathrm{a}$ & 0.03 \\
\hline QualWeb & 83 & 27 & 6 & 6.9 & 12.4 & 0 & 0 & 0 & $\mathrm{n} / \mathrm{a}$ & $\mathrm{n} / \mathrm{a}$ & 2 \\
\hline WAVE & 68 & 70 & 16 & 8.5 & 18.5 & 0 & 0 & 0 & $\mathrm{n} / \mathrm{a}$ & $\mathrm{n} / \mathrm{a}$ & 0.1 \\
\hline
\end{tabular}

state-of-the-art approaches. For each subject web page, we considered a detection to be correct (i.e., a true-positive) if KAFE indicated the page contained a KAF of a type and we had previously determined that the page contained a KAF of that type. We calculated false-positives, true-negatives, and false-negatives in an analogous way.

The four state-of-the-art tools we compared against each had their own way of reporting detections, so we normalized their results so they could be compared against KAFE. QualWeb and WAVE target several different types of accessibility related failures, such as low color contrast and missing alternative texts, therefore they generate many issue reports unrelated to KAFs. For these two tools, we considered reports with any mention of keyboard accessibility issues as a KAF detection for the corresponding web page. tabindex-counter scans and reports a ratio (0 to $1(100 \%)$ ) for a web page based on the number of interactive elements that have valid tabindex and ARIA role attributes [15, 33]. We considered any ratio less than $100 \%$ to be a detection for the page. aria-check uses behaviour-based acceptance tests to evaluate a page's dynamic behaviour with respect to accessibility requirements through 23 pre-defined usage scenario test cases. We considered the failure of any of these tests to constitute a detection. For all four tools, our mechanism for recognizing a detection was consistent with the tool's intended usage and resulted in the most favorable accuracy scores for the tool. The result of RQ1 is shown in Table 1. Columns "DEP" and "DER" show each tool's detection precision and recall for each type of KAFs.

To answer RQ2, we measured the ability of KAFE to accurately identify the faulty element(s) responsible for the KAF. For this RQ, we only compared KAFE against QualWeb and WAVE since tabindexcounter and aria-check do not provide localization information. To measure localization quality, we used the effort metric frequently used in the fault localization communities $[37,53]$ by calculating the expected number of elements a developer, using the output of each tool, would have to examine to find the fault. This metric reflects an assumption about developers' behavior and provides a normalization that allows us to quantify and relatively compare results.

To calculate KAFE's localization quality for subjects containing only a single KAF, we directly used the rank of the faulty edge in the ranked list of edges produced as the output of the localization algorithms. For subjects containing multiple failures, we calculated the rank of each faulty edge using the methodology proposed by Jones and colleagues [53]. This methodology reports the rank of the first faulty element that appears in the result set, simulates the fix of that fault, and then reruns the localization analysis to get the ranking of the next highest fault. The intuition behind using this methodology is that it approximates the workflow of a developer who scans the results, fixes a fault, and then reruns the analysis to see if any more faults remain.

To calculate the localization quality of the results generated by QualWeb and WAVE, we employed a slightly different process. This is necessitated by the fact that neither tool ranks the reported HTML elements. Therefore, we used a methodology proposed by Alameer and colleagues [37]. This technique approximates an average rank under the assumption that the faulty element is uniformly distributed in an unordered set. For a single fault, the average rank is, on average, half the size of the unordered set. In the case of multiple faults, this generalizes to a linear search for $k$ items (faults) in an unordered set of size $n$ where the distribution of the $k$ items is uniformly random. The equation for calculating this value is $(n+1) /(k+1)$. Lastly, since both tools return a set of HTML elements, as opposed to edges, we considered a localization to be successful if the tool identified a node corresponding to either the source or target of the faulty edge. In Table 1, column “\#LOR" shows the localization recall of each tool, which is the average percentage of output sets returned that contained the faulty element in any rank. Columns "\#MEF" and "\#AEF" show the median and average number of elements needed to be examined to find the KAFs for each tool.

To answer RQ3, we measured the running time of the five tools on each subject web page. For KAFE, the running time included the time to start the tool, load the browser, build the models, and compute detection and localization results (Algorithms 1 to 4). For the other four tools, the running time included the time to run the tool on a subject until the output of the tool was displayed. For QualWeb, this meant the time to submit a subject link for analysis until the result page was displayed. For WAVE, it included the time from the browser extension was clicked until the results were displayed. For tabindex-counter and aria-check, this included the time of loading the page, executing the tool's unit test codes, and displaying the result. The results of RQ3 are shown in Table 1. For each tool, the "Average" column shows the average running time.

\subsection{Discussion of Results}

For detecting IAFs, the results in Table 1 shows that KAFE was able to detect IAFs in the subject applications with high accuracy. In terms of precision, only tabindex-counter performed better; however its recall was only $39 \%$. From examining this result, we concluded that while using tabindex and role attributes to detect IAFs was a very precise approach, it did not detect all of the different ways KAFs could occur. In terms of recall, only aria-check matched the recall of KAFE for IAFs. In our examination of aria-check's results, we observed that 42 subjects failed all of its 23 test cases and all of the subjects failed at least one of the test cases. This included 
subjects that we had verified as free of IAFs. This result indicates that failures to conform to ARIA markup structure are not sufficient to indicate the presence of IAFs. Lastly, none of the approaches, except for KAFE were able to detect any of the KTFs. The keyboard traps were undetectable by the other tools because they focused on examining DOM based properties, but KTFs represent runtime behavior that is undetectable by examining the DOM.

In terms of the quality of the localization, KAFE performed significantly better than the other two approaches. One of the key results that show this is the localization recall (LOR). For IAFs, this metric shows that the HTML elements outputted by QualWeb contained only $6 \%$ of the faults and WAVE contained only $15 \%$ of the faults. In contrast, KAFE's output contained $94 \%$ of the faults. Significantly, the results of median rank (MEF) and average rank (AEF) show that KAFE was able to consistently highly rank the faulty elements. In fact, across all subjects, KAFE ranked the faulty edge in the top three for $35 \%$ of the faults, and in top ten for $76 \%$ of the faults. For KTFs, the localization quality was also high, but it was not possible to compare against other approaches since none of them could detect (and therefore localize) KTFs.

We investigated the results in more detail to understand the cases in which our approach did not correctly detect or localize. For detection, we found our approach detected two false-positive IAFs and one false-positive KTF. For the IAFs, the developers implemented section headings using non-accessible links. Based on our observations, these non-accessible links were supposed to be accessible but in this context, they offered no interactivity to the web page so we did not consider them to be true failures. Based on our experience, using links for section headings is an unusual implementation technique. For the KTF, the false-positive occurred on a captcha component. In the live web page, there was no trap. However, during our experiment, a trap occurred because our web proxy did not correctly cache this captcha component due to security protocols. For localization, our approach was unable to localize five IAFs due to Firefox's implementation of the DOM Mutation Observer Web API [14] not being able to differentiate some specific sub-states under mouse hover, which meant that they were not included in the oracle PCNFG. Our approach was also unable to localize three KTFs because in those particular cases, the developer implemented dropdown boxes that visually overlaid each other. This caused the Selenium WebDriver to be unable to interact with the dropdown in which these additional traps were located. In turn, this meant our approach could not build KNFG edges originating from these dropdown boxes.

The runtime of KAFE was significantly slower than the other approaches. However, in absolute terms the average amount of time needed by KAFE, about twenty minutes, is not a significant amount of time. Furthermore, this higher time cost is offset by its much higher precision and recall than the other approaches. We analyzed the runtime breakdown of each individual step in KAFE in detail and found that over $99 \%$ of the total time was spent on modeling and building the graphs. Specifically, the time spent on building the $\mathrm{KNFG}^{*}$ and $\mathrm{PCNFG}^{*}$ averaged 9.9 minutes and 9.2 minutes, respectively. The average time for KAF detection was 0.8 seconds and for KAF localization it was 3 seconds. The average detection and localization time for IAFs was 1.1 seconds versus 2.7 seconds for KTFs.
GROUPON

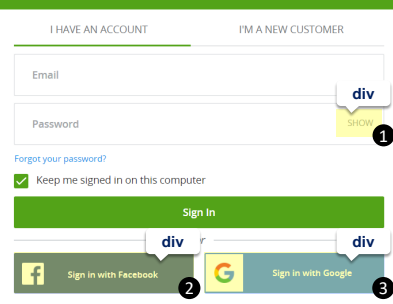

(a) Groupon page where the "Show" password button (1) is not reachable (focusable), and the Facebook and Google log-in buttons $(2,8)$ are reachable (focusable) but not actionable by the keyboard.

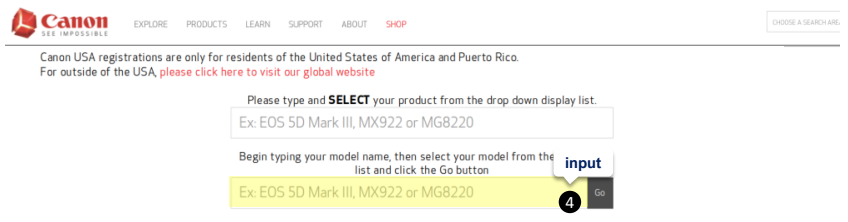

(b) Canon product registration page where the keyboard focus is trapped on the product model input box (4).

\section{Figure 4: Real-world examples of KAFs}

5.4.1 The impact of KAFs. The impact of the KAFs that we found in our subjects was generally quite severe. For IAFs, often core functionalities of the web page were unavailable to keyboard-based users. For example, they would be unable to log in via Google or Facebook's external authentication (e.g., Figure 4a), unable to retrieve their password, unable to change their language/locality preference, or sometimes unable to use the "Like" Facebook plugin. The impact for KTFs was even more dramatic. Users would get stuck when entering data into web forms with auto-tabbing scripts, preventing them from moving backwards to make corrections. This is particularly troublesome for blind users that must move backwards through the fields to verify that they correctly entered the numbers via screen readers. When stuck, they would be forced to refresh the page, or close the browser and risk losing all of the form information entered.

5.4.2 The root causes of KAFs. We analyzed the subject web pages to identify root causes of IAFs and found several reoccurring patterns. (1) Custom UI controls based on $<$ div $>$, $<$ span $>$, or $<\mathrm{li}>$ where developers would implement mouse event handlers but not keyboard handlers. We found that 37 out of 455 buttons and 22 out of 58 dropdown-lists across our subject pool were inaccessible due to their inability to receive keyboard focus. We also found 34 instances where $\mathrm{a}<\mathrm{a}>$ was used to trigger JavaScript instead of having an "href" attribute. As a result, these elements were not able to receive keyboard focus [34]. We found two instances where the developer explicitly assigned a tabindex of 0 to make a custom widget focusable but yet, without defining any keyboard event handlers, the controls were not actionable. (2) Actions triggered by mouse hovering. Overall, a remarkably high 35 of 49 menus implemented to expand when a mouse hovered over them were inaccessible. Roughly a third of those could not receive focus, and most of the others that could receive focus could not expand via a keyboard action. This problem also appeared when tooltips were 
implemented using custom mechanisms. (3) Overriden standard control elements. We found six cases of checkbox/radio-button whose operations were delegated to a programmatically associated $<$ label $>$ [32]. Clicking on the <label $>$ indirectly activates the control. However, for aesthetics, the developers made these control hidden and styled its <label> 's CSS to make it appear to be a custom checkbox/radio-button. These inaccessible implementations assume that users would be able to "click" on the <label $>$ to tick the associated control.

For root causes of KTFs, we similarly found several reoccurring patterns. (1) Auto-advance during data entry in web forms. Over half of the of the KTFs we found were due to mistakes in implementing this functionality. (2) Customized widgets bound to scripts that altered the focus or the layout of the UI. Often unintended side effects of this alteration would lead to the KTF. (3) Text input boxes with overriden keydown and keypress events. Typically, these would be used to auto-complete data entered into search boxes or to filter the contents of dropdown lists as users entered text (e.g., Figure 4b). Mistakes in the implementation of these handlers resulted in keyboard users being unable to navigate away from the input box.

5.4.3 Limitations. The primary cause of inaccuracy in our approach was when Selenium WebDriver was unable to interact with some elements that it considers "NotInteractable" [30] or when the DOM was considered "Stale" [4]. These situations occur when elements are obstructed by others, or when the DOM is spontaneously altered by AJAX calls that were not triggered via our automated interaction (e.g., events automatically loaded via a timer, a slider/carousel that constantly loads persistent streams, or asynchronous calls that implement infinite scrolling to load and populate data). We believe that further improvements in web page capture and replay techniques would allow for this behavior to be more reliably controlled for during analysis.

\subsection{Threats to Validity}

A potential threat to external validity is that our tool is implemented using Selenium's FirefoxDriver but there could be potential discrepancies in keyboard navigation behaviors between different web browsers that causes cross-browser behavioral inconsistencies. However, it is important to note that the KAF ground-truth would therefore also vary. These browser-specific KAFs could be easily detected by systematically running KAFE using different Selenium WebDrivers for different browsers.

A potential threat to criterion validity is that both the KAFs (ground-truth) and faults were identified by the authors. In terms of mitigating this threat, it is important to note that the success criteria in WCAG are, by design, defined in such a way as to make it possible to objectively determine when a failure has occurred [1]. In addition, we followed best practices defined in [10, 19, 26] and there was no disagreement between the authors in terms of what was classified as a IAFs or KTFs. The failures are also defined in terms of behaviors of specific elements of a UI, which makes it possible to objectively determine the faulty element that should be identified by KAFE. As further validation, we performed a follow up investigation of the KAFs we identified in our study. Since we began the research project, 12 of the 40 subjects had been repaired by the developers. We reached out via the website's contact forms to the remaining 28 to report our findings. Fifteen acknowledged our report, and three of them reported that they were addressing the KAFs.

\section{RELATED WORK}

Guideliner [55] is a tool that assesses web UI element conformance to a predefined set of usability guidelines. It focuses on visual characteristics of web UIs, such as the position of elements on the screen, the distance between the elements, and the length of scrolling. VizAssert $[58,59]$ uses formal verification methods to attempt detection and repair on inaccessible layout properties. Both approaches focus on accessibility relating to layout properties and are not capable of interacting with the PUT to expose KAFs.

AXERAY [42] is an automated approach that infers semantic groupings of elements across various regions of a web page to test if these elements violate their WAI-ARIA roles' semantic structure. Research work [69] verifies ARIA specifications [46] on dynamic content and UI components to detect interactive accessibility issues. Although ARIA is useful in providing custom attributes via the browser's Accessibility API, it alone does not ensure these interactable control elements are properly scripted to be accessible. Work done by Watanabe et al. evaluates various dynamic UI elements that can cause AT accessibility issues. They characterize complex custom widgets and navigation elements such as dropdown menus [40], tab widgets [70], menu popups, date picker, [56], slideshows and Carousels widgets [44] to ensure they behave according to accessibility standards. Although useful for inspiring best practices for implementing IAF conformance, these ad-hoc approaches cannot be generalized to handle keyboard on a more diverse scale.

Many techniques focus on mobile accessibility issues and their conformance measures based on violations [60, 61, 65, 72-74], particularly, [39, 64, 72] developed accessibility checkers to identify and categorize the identified issues. While the majority of this research is related to mobile accessibility failure detection, research by Zhang et al. focus on repairing these failures [61, 73, 74] as well as other deep learning [45] and search-based [38] repair. Latte [62] is another technique that automatically executes use cases using assistive services to detect accessibility failures. However, these approaches only work on Android apps, not web apps.

\section{CONCLUSION}

Poorly designed web applications create keyboard accessibility barriers that exclude people with disabilities from interacting with its functionalities. In this paper, we presented a novel approach for automatically detecting and localizing KAFs in web pages. The evaluation showed that our approach could detect and localize keyboard failures with high precision and recall. We believe these results are positive and show that our approach could help developers to debug KAFs in web applications.

\section{ACKNOWLEDGMENTS}

This work was supported by National Science Foundation under Grant No. 2009045. 


\section{REFERENCES}

[1] 2016. W3C: Techniques and Failures for Web Content Accessibility Guidelines 2.0. https://www.w3.org/TR/WCAG20-TECHS/. Updated: 2016-10-07.

[2] 2017. The Section 508 Refresh and What It Means for Higher Education. https://er..edu/articles/2017/12/the-section-508-refresh-and-what-itmeans-for-higher-education. Updated: 2017-12-04.

[3] 2019. The Internet is unavailable. https://nucleusresearch.com/research/single/ the-internet-is-unavailable/

[4] 2019. Selenium: Stale Element Reference Exception . https://www.selenium.dev/ exceptions/\#: :text=A\%20stale\%20element. Accessed: 2021-02-15.

[5] 2019. W3C WAI: Introduction to Web Accessibility. https://www.w3.org/WAI/ fundamentals/accessibility-intro/. Updated: 2019-06-05.

[6] 2019. W3C WAI: Keyboard Compatibility. https://www.w3.org/WAI/perspectivevideos/keyboard/. Updated: 2019-01-23.

[7] 2019. Web Accessibility Evaluation Tools List. https://www.w3.org/WAI/ER/ tools/. Updated: 2016-03.

[8] 2020. Beebom: What is HTML5 and Why Has it Replaced Flash and Silverlight? https://beebom.com/what-is-html5/. Updated: 2020-12-24.

[9] 2020. COVID-19 Pushes Commerce Online, Making ADA Website Compliance More Important Than Ever. https://www.law.com/legaltechnews/2020/05/19/ covid-19-pushes-commerce-online-making-ada-website-compliance-moreimportant-than-ever/. Updated: 2020-05-19.

[10] 2020. CSUN Universal Design Center: Web Accessibility Criteria - Keyboard Accessibility. https://www.csun.edu/universal-design-center/web-accessibilitycriteria-keyboard-accessibility. Accessed: 2020-07-03.

[11] 2020. DOM Living Standard. https://dom.spec.whatwg.org/\#concept-tree-order. Accessed: 2020-08-28.

[12] 2020. Keyboard Accessible: Understanding Guideline 2.1. https://www.w3.org/ TR/UNDERSTANDING-WCAG20/keyboard-operation.html

[13] 2020. MDN Web Docs: :hover CSS pseudo-class. https://developer.mozilla.org/enUS/docs/Web/CSS/:hover. Accessed: 2020-08-16.

[14] 2020. MDN Web Docs: MutationObserverInit.childList. https: //developer.mozilla.org/en-US/docs/Web/API/MutationObserverInit/childList. Accessed: 2020-08-16.

[15] 2020. MDN Web Docs: Using ARIA: Roles, states, and properties. https:/ developer.mozilla.org/en-US/docs/Web/Accessibility/ARIA/ARIA_Techniques. Accessed: 2020-08-18.

[16] 2020. mitmproxy: a free and open source interactive HTTPS proxy. https: //mitmproxy.org/. Accessed: 2020-08-16.

[17] 2020. Moz's list of the most popular 500 websites on the internet. https:// moz.com/top500. Accessed: 2020-08-25.

[18] 2020. U.S. GSA 18F Accessibility Guide: Keyboard access. https:// accessibility.18f.gov/keyboard/. Accessed: 2020-08-25.

[19] 2020. UW: Designing for Keyboard Accessibility. https://www.washington.edu/ accessibility/checklist/keyboard/. Accessed: 2020-08-16.

[20] 2020. W3C: How People with Disabilities Use the Web. https://www.w3.org/ WAI/people-use-web/. Accessed: 2020-08-27.

[21] 2020. W3C: Keyboard Accessible - Understanding Guideline 2.1. https: //www.w3.org/TR/UNDERSTANDING-WCAG20/keyboard-operation.html. Accessed: 2020-08-27.

[22] 2020. W3C: UI Events - Mouse Event Types. https://www.w3.org/TR/uievents/ \# events-mouse-types. Accessed: 2020-08-28.

[23] 2020. W3C: User Agent Accessibility Guidelines (UAAG) Overview. https: //www.w3.org/WAI/standards-guidelines/uaag/. Accessed: 2020-08-27.

[24] 2020. W3C: WAI-ARIA Authoring Practices 1.1 - Keyboard Interaction. https: //www.w3.org/TR/wai-aria-practices-1.1/\#keyboard-interaction. Accessed: 2020-08-28.

[25] 2020. WAVE Web Accessibility Evaluation Tool. https://wave.webaim.org/. Accessed: 2020-08-25.

[26] 2020. WebAIM: Keyboard Accessibility - Keyboard Testing. https://webaim.org/ techniques/keyboard/\#testing. Accessed: 2020-07-03.

[27] 2020. Wikipedia: Bridge (graph theory). https://en.wikipedia.org/wiki/ Bridge_(graph_theory). Accessed: 2020-08-28.

[28] 2020. World Health Organization: World Report on Disability. https:// www.who.int/disabilities/world_report/2011/report/en/. Accessed: 2020-0702.

[29] 2021. AUTO TAB HTML INPUT FIELDS. https://bartamediagroup.com/autotab-html-input-fields/. Updated: 2016-08-21.

[30] 2021. GitHub Issues: Element Not Interactable Exception in Selenium . https: //github.com/SeleniumHQ/selenium/issues/4314.

[31] 2021. KAFE Project Web Site. https://sites.google.com/usc.edu/kafe/home. Updated: 2021-06-16.

[32] 2021. MDN Web Docs: <label> - for. https://developer.mozilla.org/en-US/docs/ Web/HTML/Element/label\#attr-for. Accessed: 2021-06-18.

[33] 2021. MDN Web Docs: tabindex. https://developer.mozilla.org/en-US/docs/Web/ HTML/Global_attributes/tabindex. Updated: 2021-01-16.

[34] 2021. Techniques for WCAG 2.1: Making actions keyboard accessible by using the onclick event of anchors and buttons. https://www.w3.org/WAI/WCAG21/
Techniques/client-side-script/SCR35.html. Accessed: 2021-01-19.

[35] 2021. The Selenium Browser Automation Project: WebDriver - Keyboard. https: //www.selenium.dev/documentation/en/webdriver/keyboard/. Accessed: 202101-14.

[36] Julio Abascal, Myriam Arrue, Inmaculada Fajardo, and Nestor Garay. 2006. An Expert-Based Usability Evaluation of the EvalAccess Web Service. In HCI related papers of Interacción 2004, Raquel Navarro-Prieto and Jesús Lorés Vidal (Eds.). Springer Netherlands, Dordrecht, 1-17. https://doi.org/10.1007/1-4020-4205-1 1

[37] A. Alameer, S. Mahajan, and W. G. J. Halfond. 2016. Detecting and Localizing Internationalization Presentation Failures in Web Applications. In 2016 IEEE International Conference on Software Testing, Verification and Validation (ICST). 202-212.

[38] Ali S. Alotaibi, Paul T. Chiou, and William G. J. Halfond. 2021. Automated Repair of Size-Based Accessibility Issues in Mobile Applications. In Proceedings of the 36th IEEE/ACM International Conference on Automated Software Engineering (Virtual Event, Australia) (ASE '21). Association for Computing Machinery, New York, NY, USA.

[39] Abdulaziz Alshayban, Iftekhar Ahmed, and Sam Malek. 2020. Accessibility Issues in Android Apps: State of Affairs, Sentiments, and Ways Forward. In Proceedings of the ACM/IEEE 42nd International Conference on Software Engineering (Seoul, South Korea) (ICSE '20). Association for Computing Machinery, New York, NY, USA, 1323-1334. https://doi.org/10.1145/3377811.3380392

[40] Humberto Lidio Antonelli, Rodrigo Augusto Igawa, Renata Pontin De Mattos Fortes, Eduardo Henrique Rizo, and Willian Massami Watanabe. 2018. DropDown Menu Widget Identification Using HTML Structure Changes Classification. ACM Transactions on Accessible Computing 11, 2 (June 2018), 10:1-10:23. https: //doi.org/10.1145/3178854

[41] Humberto Lidio Antonelli, Leonardo Sensiate, Willian Massami Watanabe, and Renata Pontin de Mattos Fortes. 2019. Challenges of automatically evaluating rich internet applications accessibility. In Proceedings of the 37th ACM International Conference on the Design of Communication (SIGDOC '19). Association for Computing Machinery, Portland, Oregon, 1-6. https://doi.org/10.1145/3328020.3353950

[42] Mohammad Bajammal and Ali Mesbah. 2021. Semantic Web Accessibility Testing via Hierarchical Visual Analysis. In 2021 IEEE/ACM 43rd International Conference on Software Engineering (ICSE). 1610-1621. https://doi.org/10.1109/ ICSE43902.2021.00143

[43] Peter Blanck. [n.d.]. ADA Title III and Web Equality: Litigation Begins. eQuality ([n. d.]), 81-104. https://doi.org/10.1017/cbo9781107280151.009

[44] Alex Chen, Simon Harper, Darren Lunn, and Andy Brown. 2013. Widget Identification: A High-Level Approach to Accessibility. World Wide Web 16 (01 2013), 73-89. https://doi.org/10.1007/s11280-012-0156-6

[45] Jieshan Chen, Chunyang Chen, Zhenchang Xing, X. Xu, Liming Zhu, Guoqiang $\mathrm{Li}$, and J. Wang. 2020. Unblind Your Apps: Predicting Natural-Language Labels for Mobile GUI Components by Deep Learning. ArXiv abs/2003.00380 (2020).

[46] Joanmarie Diggs, Shane McCarron, Michael Cooper, Richard Schwerdtfeger, and James Craig. [n.d.]. Accessible Rich Internet Applications (WAI-ARIA) 1.1. https://www.w3.org/TR/wai-aria/

[47] Nádia Fernandes, Daniel Costa, Sergio Neves, Carlos Duarte, and Luís Carriço. 2012. Evaluating the accessibility of rich internet applications. In Proceedings of the International Cross-Disciplinary Conference on Web Accessibility (W4A '12). Association for Computing Machinery, Lyon, France, 1-4. https://doi.org/ 10.1145/2207016.2207019

[48] José L. Fuertes, Emmanuelle Gutiérrez, and Loïc Martínez. 2011. Developing Hera-FFX for WCAG 2.0. In Proceedings of the International Cross-Disciplinary Conference on Web Accessibility (Hyderabad, Andhra Pradesh, India) (W4A '11). Association for Computing Machinery, New York, NY, USA, Article 3, 9 pages. https://doi.org/10.1145/1969289.1969294

[49] Greg Gay and Cindy Qi Li. 2010. AChecker: Open, Interactive, Customizable, Web Accessibility Checking. In Proceedings of the 2010 International Cross Disciplinary Conference on Web Accessibility (W4A) (Raleigh, North Carolina) (W4A '10). Association for Computing Machinery, New York, NY, USA, Article 23, 2 pages. https://doi.org/10.1145/1805986.1806019

[50] Becky Gibson and Richard Schwerdtfeger. 2005. DHTML accessibility: solving the JavaScript accessibility problem. In Proceedings of the 7th international ACM SIGACCESS conference on Computers and accessibility (Assets '05). Association for Computing Machinery, Baltimore, MD, USA, 202-203. https://doi.org/10.1145/ 1090785.1090830

[51] Teresa D. Gilbertson and Colin H. C. Machin. 2012. Guidelines, icons and marketable skills: an accessibility evaluation of 100 web development company homepages. In Proceedings of the International Cross-Disciplinary Conference on Web Accessibility (W4A '12). Association for Computing Machinery, Lyon, France, 1-4. https://doi.org/10.1145/2207016.2207024

[52] Wan Abdul Rahim Wan Mohd Isa, Ahmad Iqbal Hakim Suhaimi, Nadhirah Ariffrn, Nurul Fatimah Ishak, and Nadilah Mohd Ralim. 2016. Accessibility evaluation using Web Content Accessibility Guidelines (WCAG) 2.0. In 2016 4th International Conference on User Science and Engineering (i-USEr). 1-4. https://doi.org/10.1109/ IUSER.2016.7857924 
[53] James A. Jones, James F. Bowring, and Mary Jean Harrold. 2007. Debugging in Parallel. In Proceedings of the 2007 International Symposium on Software Testing and Analysis (London, United Kingdom) (ISSTA '07). Association for Computing Machinery, New York, NY, USA, 16-26. https://doi.org/10.1145/1273463.1273468

[54] Jonathan Lazar. 2019. The potential role of U.S. consumer protection laws in improving digital accessibility for people with disabilities. U. Pa. JL \& Soc. Change 22 (2019), 185.

[55] Jevgeni Marenkov, Tarmo Robal, and Ahto Kalja. 2018. Guideliner: a Tool to Improve Web UI Development for Better Usability. In Proceedings of the 8th International Conference on Web Intelligence, Mining and Semantics (WIMS '18) Association for Computing Machinery, Novi Sad, Serbia, 1-9. https://doi.org/ $10.1145 / 3227609.3227667$

[56] Valentyn Melnyk, Vikas Ashok, Valentyn Melnyk, Yury Puzis, Yevgen Borodin Andrii Soviak, and I. V. Ramakrishnan. 2015. Look Ma, no ARIA: generic accessible interfaces for web widgets. In Proceedings of the 12th Web for All Conference (W4A '15). Association for Computing Machinery, Florence, Italy, 1-4. https: //doi.org/10.1145/2745555.2746666

[57] Ali Mesbah, Arie van Deursen, and Stefan Lenselink. 2012. Crawling Ajax-Based Web Applications Through Dynamic Analysis of User Interface State Changes. ACM Trans. Web 6, 1, Article 3 (March 2012), 30 pages. https://doi.org/10.1145/ 2109205.2109208

[58] Pavel Panchekha, Michael D. Ernst, Zachary Tatlock, and Shoaib Kamil. 2019. Modular verification of web page layout. Proceedings of the ACM on Programming Languages 3, OOPSLA (Oct. 2019), 151:1-151:26. https://doi.org/10.1145/3360577

[59] Pavel Panchekha, Adam T. Geller, Michael D. Ernst, Zachary Tatlock, and Shoaib Kamil. 2018. Verifying that web pages have accessible layout. In Proceedings of the 39th ACM SIGPLAN Conference on Programming Language Design and Implementation (PLDI 2018). Association for Computing Machinery, Philadelphia, PA, USA, 1-14. https://doi.org/10.1145/3192366.3192407

[60] Anne Ross, Xiaoyi Zhang, James Fogarty, and Jacob O. Wobbrock. 2020. An Epidemiology-Inspired Large-Scale Analysis of Android App Accessibility. ACM Trans. Access. Comput. 13, 1, Article 4 (April 2020), 36 pages. https://doi.org/ $10.1145 / 3348797$

[61] Anne Spencer Ross, Xiaoyi Zhang, James Fogarty, and Jacob O. Wobbrock 2018. Examining Image-Based Button Labeling for Accessibility in Android Apps Through Large-Scale Analysis. In Proceedings of the 20th International ACM SIGACCESS Conference on Computers and Accessibility (Galway, Ireland) (ASSETS '18). ACM, New York, NY, USA, 119-130. https://doi.org/10.1145/ 3234695.3236364

[62] Navid Salehnamadi, Abdulaziz Alshayban, Jun-Wei Lin, Iftekhar Ahmed, Stacy Branham, and Sam Malek. 2021. Latte: Use-Case and Assistive-Service Driven Automated Accessibility Testing Framework for Android. Association for Computing Machinery, New York, NY, USA. https://doi.org/10.1145/3411764.3445455

[63] Antonio Giovanni Schiavone and Fabio Paternò. 2015. An extensible environment for guideline-based accessibility evaluation of dynamic Web applications. Universal Access in the Information Society 14 (03 2015), 111-132. https://doi.org/10.1007/s10209-014-0399-3

[64] Camila Silva, Marcelo Medeiros Eler, and Gordon Fraser. 2018. A Survey on the Tool Support for the Automatic Evaluation of Mobile Accessibility. In Proceedings of the 8th International Conference on Software Development and Technologies for Enhancing Accessibility and Fighting Info-exclusion (Thessaloniki, Greece) (DSAI 2018). ACM, New York, NY, USA, 286-293. https://doi.org/10.1145/ 3218585.3218673

[65] C. Vendome, D. Solano, S. Liñán, and M. Linares-Vásquez. 2019. Can Everyone use my app? An Empirical Study on Accessibility in Android Apps. In 2019 IEEE International Conference on Software Maintenance and Evolution (ICSME). 41-52.

[66] Willian Massami Watanabe, Ana Luiza Dias, and Renata Pontin De Mattos Fortes. 2015. Fona: Quantitative Metric to Measure Focus Navigation on Rich Internet Applications. ACM Transactions on the Web 9, 4 (Sept. 2015), 20:1-20:28. https: //doi.org/10.1145/2812812

[67] Willian Massami Watanabe, Renata P. Fortes, and Ana Luiza Dias. 2017. Acceptance Tests for Validating ARIA Requirements in Widgets. Univers. Access Inf. Soc. 16, 1 (March 2017), 3-27. https://doi.org/10.1007/s10209-015-0437-9

[68] Willian Massami Watanabe, Renata P. M. Fortes, and Ana Luiza Dias. 2012. Using acceptance tests to validate accessibility requirements in RIA. In Proceedings of the International Cross-Disciplinary Conference on Web Accessibility (W4A '12). Association for Computing Machinery, Lyon, France, 1-10. https://doi.org/ $10.1145 / 2207016.2207022$

[69] Willian Massami Watanabe, Renata P. M. Fortes, and Ana Luiza Dias. 2017. Acceptance tests for validating ARIA requirements in widgets. Universal Access in the Information Society 16, 1 (March 2017), 3-27. https://doi.org/10.1007/s10209015-0437-9

[70] Willian Massami Watanabe, Rafael José Geraldo, and Renata Pontin de Mattos Fortes. 2014. Keyboard navigation mechanisms in tab widgets: an investigation on ARIA's conformance. In Proceedings of the 29th Annual ACM Symposium on Applied Computing (SAC '14). Association for Computing Machinery, Gyeongju, Republic of Korea, 721-726. https://doi.org/10.1145/2554850.2554947

[71] B. Wentz, P.T. Jaeger, and J.C. Bertot. 2015. Accessibility for Persons with Disabilitie and the Inclusive Future of Libraries. Emerald Group Publishing Limited. https: //books.google.com/books?id=tcNOCwAAQBAJ

[72] Shunguo Yan and P. G. Ramachandran. 2019. The Current Status of Accessibility in Mobile Apps. ACM Trans. Access. Comput. 12, 1, Article 3 (Feb. 2019), 31 pages. https://doi.org/10.1145/3300176

[73] Xiaoyi Zhang, Anne Spencer Ross, Anat Caspi, James Fogarty, and Jacob O. Wobbrock. 2017. Interaction Proxies for Runtime Repair and Enhancement of Mobile Application Accessibility. In Proceedings of the 2017 CHI Conference on Human Factors in Computing Systems (Denver, Colorado, USA) (CHI '17). ACM, New York, NY, USA, 6024-6037. https://doi.org/10.1145/3025453.3025846

[74] Xiaoyi Zhang, Anne Spencer Ross, and James Fogarty. 2018. Robust Annotation of Mobile Application Interfaces in Methods for Accessibility Repair and Enhancement. In Proceedings of the 31st Annual ACM Symposium on User Interface Software and Technology (Berlin, Germany) (UIST '18). ACM, New York, NY, USA, 609-621. https://doi.org/10.1145/3242587.3242616 